\title{
Comparison of Different Edge Detections and Noise Reduction on Ultrasound Images of Carotid and Brachial Arteries Using a Speckle Reducing Anisotropic Diffusion Filter
}

\author{
Mehravar Rafati ${ }^{1}$; Masoud Arabfard ${ }^{2, *} ;$ Mehrdad Rafati-Rahimzadeh $^{3}$ \\ ${ }_{1}^{1}$ Department of Medical Physics, Faculty of Paramedical Sciences, Kashan University of Medical Sciences, Kashan, IR Iran \\ ${ }^{2}$ Department of Health Information Management and Technology, Faculty of Paramedical Sciences, Kashan University of Medical Sciences, Kashan, IR Iran \\ ${ }^{3}$ Department of Nursing, Babol University of Medical Sciences, Babol, IR Iran \\ ${ }^{*}$ Corresponding Author: Masoud Arabfard, Department of Health Information Management and Technology, Faculty of Paramedical Sciences, Kashan University of Medical Sciences, \\ Kashan, IR Iran. Tel: +98-9132649405, E-mail: arabfard-ma@kaums.ac.ir
}

Received: September 5, 2013; Revised: November 11, 2013; Accepted: April 21, 2014

\begin{abstract}
Background: Common carotid artery (CCA) ultrasound with measurement of intima-media thickness (IMT) is a safe and noninvasive technique for assessing subclinical atherosclerosis and determining cardiovascular risks. Moreover, the pattern of wall thickening in the brachial artery (BA) is rather diffuse compared to the carotid artery and may be a more sensitive indicator of long-term systemic exposure to risk factors. Therefore noninvasive evaluation of mechanical parameters changes of both arteries has gained the attention of researchers.

Objectives: The aim of this study was to compare different edge detection techniques with speckle reducing anisotropic diffusion (SRAD) de-noising filter in ultrasound images of both arteries.

Patients and Methods: In a cross-sectional design, an examination was performed on ten men with mean age of $40 \pm 5$ years from September 2012 to March 2013 through random sampling. An ultrasonic examination of the left CCA and BA was performed. The program was designed in the MATLAB software to extract consecutive images in JPEG format from the AVI. Another program was designed in the MATLAB software to apply regions of interest(ROI) on the IMT of the posterior wall of common carotid and brachial arteries. Next, different edge detections and SRAD filter were applied to the ROI, separately. Finally, the program measured mean-squared error (MSE) and peak signal to noise ratio (PSNR).

Results: The lowest values of MSE and highest values of PSNR were achieved by Canny edge detection with de-noising SRAD filter for IMT of left CCA and BA in 90 frames.

Conclusions: Based on the result, by measuring the MSE and PSNR, this study showed Canny edge detection with SRAD filter is better than other edge detections in terms of speckle suppression and details preservation in CCA and BA ultrasound images.
\end{abstract}

Keywords:Carotid Artery; Brachial Artery; Immature Thymocyte-Specific Antigen (IMT); Edge Detection; De-Noising Filter

\section{Background}

Ultrasound imaging is broadly used to show carotid, femoral, brachial and other peripheral arteries. There are several important benefits of using ultrasound in comparison to other techniques. Most importantly, B-mode ultrasound imaging is non-invasive and allows real time conception of arterial morphology, which is not currently possible with any other imaging tool (1). Furthermore, the non-invasive nature provided by B-mode ultrasound imaging and its low cost has allowed the use of this technique for more clinical studies, which show a major relationship between carotid intima-media thickness (IMT) and cardiovascular disease (CVD) (2). Ultrasonic B-mode images from IMT of common carotid artery (CCA) are used broadly as a measure of atherosclerosis and in studies on atherosclerosis as the determinant of CVD. Carotid IMT has been shown to be related to cardiovascular risk factors, current CVD, and atherosclerosis in the peripher- al coronary, femoral and brachial arteries (3). Ultrasound images edge detection is important for recognition of IMT in CCA and brachial artery (BA). Generally, edge is detected according to algorithms such as Sobel, Roberts, Prewitt, Canny, and LOG (Laplacian of Gaussian) operators (4), yet in theory they consist of high pass filtering, which are not appropriate for noise ultrasound image edge detection because noise and edge belong to the range of high frequency $(5,6)$. Naturally, an ultrasound image includes more noise content, especially speckle noise, than any other imaging modality (6). Speckle is the artifact caused by interference of energy from randomly distributed scattering objects which reduces image resolution and contrast and blurs essential details. Therefore, speckle noise suppression is an important requirement whenever ultrasound imaging is used $(6,7)$. Speckle is not a kind of additive noise, it is instead a form of multiplicative noise (8). 
Rafati M et al.

Traditional speckle removal filters, like the Lee filter and Frost filter have greater restrictions in edge and characteristics preservation (9). A noise reduction filter such as conventional anisotropic diffusion is not appropriate for speckle suppression (8). The conventional anisotropic is not appropriate for multiplicative noise-including speckle and it is instead effective for additive noise. Yongjian et al. first introduced the speckle reducing anisotropic diffusion (SRAD) filter (9). The SRAD filter joins both the additive noise reduction anisotropic diffusion filtering process and the adaptive speckle (multiplicative noise) filtering process (9). It makes the generation of image scale area possible (a set of filtered images that alter from fine to coarse) without bias and with filter window size and shape (9). The SRAD filter not only protects edges but also enhances edges by eliminating diffusion across edges and allowing diffusion on either side of the edge (8).

\section{Objectives}

The aim of this study was to compare different edge detection techniques with SRAD de-noising filter in ultrasound images of common carotid and brachial arteries for optimum removal of noise with preservation of edges.

\section{Materials and Methods}

\subsection{Edge Detection}

The edge detection process involves small kernels that convolve with an image to estimate the first-order directional derivatives of the image brightness distribution. Kernels are pre-defined groups of edge models that match each image segment of a fixed size. The edge value is calculated by forming a matrix centered on each pixel. If the value is not smaller than a given threshold, then the pixel is categorized as an edge. All the gradientbased algorithms have kernel operators that estimate the edge strength in directions, which are orthogonal to one another, generally vertically and horizontally. The contribution of both parts are combined to give the total value of edge strength (10). Edge detection algorithms are grouped into two categories, namely, gradient operator and Laplacian operator. The gradient operator detects edge pixels by obtaining the maximum and minimum value at the first derivative level on the image. The classical gradient operators selected in this work are Sobel, Prewitt, Muthukrishnan and Radha (11). Laplacian operator is a second order derivative, where the value of edge pixel at the first derivative is referred to as zero-crossing at the second derivative (11). The operators are explained below.

\subsubsection{Sobel Operator}

The Sobel operator $(11,12)$ is a discrete operator, which computes the gradient for intensity changes at each point in an image. The operator consists of a pair of $3^{*} 3$ convolution kernels as shown in equation 1 (1):

\begin{tabular}{|c|c|}
\hline$s_{x}=$ & $\begin{array}{lll}-1 & 0 & 1 \\
-2 & 0 & 2 \\
-1 & 0 & 1\end{array}$ \\
\hline$s_{y}=$ & $\begin{array}{ccc}-1 & -2 & -1 \\
0 & 0 & 0 \\
1 & 2 & 1\end{array}$ \\
\hline
\end{tabular}

Equation 1. The $3^{*} 3$ kernels for the Sobel operator (Sx and Sy are gradient components for the horizontal and vertical edge orientations). These kernels are designed to respond maximally to edges running vertically and horizontally relative to the pixel matrix; one kernel for each of the two perpendicular orientations. The gradient magnitude is extracted by: (2)

$$
|s|=\sqrt{s_{x}^{2}+s_{y}^{2}}
$$

Sx and Sy are gradient parts for the horizontal and vertical edge orientations.

Typically, an approximate magnitude is computed using: (3)

$$
|s|=|s|+s
$$

This is much faster to compute. The angle of orientation of the edge (relative to the pixel matrix) giving rise to the spatial gradient is extracted by:(4).

$$
\theta=\arctan \left(s_{y} / s_{x}\right)
$$

\subsubsection{Roberts Operator}

The Roberts operator (13) performs a simple, quick to compute, two-dimensional (2-D) spatial gradient measurement on an image. The operator consists of a pair of $2 * 2$ convolution kernels as shown in equation 5 (5).

$$
R=\begin{array}{cc}
1 & 0 \\
0 & -1
\end{array}
$$

$$
R=\begin{array}{cc}
0 & 1 \\
-1 & 0
\end{array}
$$

The $2 \times 2$ kernels for Roberts operator ( $\mathrm{Rx}$ and Ry are gradient components for the horizontal and vertical edge orientations). These kernels are designed to respond maximally to edges running at 45 to the pixel matrix; one kernel for each of the two perpendicular orientations. The kernels can be used several times in the input image, 
to create independent measurements of the gradient component in each orientation ( $\mathrm{Rx}$ and $\mathrm{Re}$ ). The gradient magnitude is extracted by:(6).

$$
|R|=\sqrt{R_{x}^{2}+R_{y}^{2}}
$$

The angle of orientation of the edge (relative to the pixel matrix) giving rise to the spatial gradient is extracted by (5):

$$
\theta=\arctan \left(\frac{R_{y}}{R_{x}}\right)-\frac{3 \pi}{4}
$$

\subsubsection{Prewitt Operator}

Prewitt operator $(11,13)$ is a discrete differentiation operator which functions similar to the Sobel operator, by computing the gradient for the image intensity function. The Prewitt edge detection operator is used for detecting vertical and horizontal edges of images (equation 6) (6).

$$
P_{x}=\begin{array}{ccc}
1 & 1 & 1 \\
0 & 0 & 0 \\
-1 & -1 & -1
\end{array}
$$

$$
P_{y}=\begin{array}{lll}
-1 & 0 & 1 \\
-1 & 0 & 1 \\
-1 & 0 & 1
\end{array}
$$

The $3 \times 3$ kernels for Prewitt operator $\left(\mathrm{P}_{\mathrm{x}}\right.$ and $\mathrm{P}_{\mathrm{y}}$ are gradient components for the horizontal and vertical edge orientations).

\subsubsection{Laplacian of Gaussian (LOG) Operator}

The Laplacian is a 2-D isotropic measure of the second spatial derivative of an image. The Laplacian of an image highlights districts of rapid intensity change and is therefore often used for edge detection. The Laplacian is often used for an image that has first been smoothed with something approximating a Gaussian-smoothing filter in order to reduce its sensitivity to noise. The operator commonly takes a single gray level image as input and produces another gray level image as output. The Laplacian $\mathrm{L}(\mathrm{x}, \mathrm{y})$ of an image with pixel intensity values I ( $\mathrm{x}$, y) is extracted by (7):

$$
L(x, y)=\frac{\partial^{2} I}{\partial x^{2}}+\frac{\partial^{2} I}{\partial x^{2}}
$$

As shown here, the input image is illustrated as a set of discrete pixels; a discrete convolution kernel can approximate the second derivative in the definition of the Laplacian. Three commonly used small kernels are shown in below equations (8):

$\begin{array}{ccc}0 & 1 & 0 \\ 1 & -4 & 1 \\ 0 & 1 & 0\end{array}$

\begin{tabular}{cccc}
\hline & & & \\
\hline 1 & 1 & 1 \\
1 & -8 & 1 \\
1 & 1 & 1 \\
\hline & & \\
-1 & 2 & -1 \\
2 & -4 & 2 \\
-1 & 2 & -1 \\
\hline
\end{tabular}

Three commonly used discrete approximations to the Laplacian filter. Since these kernels are approximating a second derivative measurement on the image, they are extremely sensitive to noise. To indicate this, the image is often Gaussian smoothed before applying the Laplacian filter. The Log function centered on zero and with Gaussian standard deviation $(\sigma)$ results in the following equation (9):

$$
\log (x, y)=-\left(\frac{1}{\pi \sigma^{4}}\right)
$$

\subsubsection{Canny Operator}

Canny edge detector first smoothes the image to remove noise and then determines the image gradient to highlight regions with high spatial derivatives. The algorithm then tracks along these districts and suppresses any pixel that is not at the maximum (non-maximum suppression). The gradient array is now more reduced by hysteresis. Hysteresis is used to chase along the remaining pixels that have not been reduced. Hysteresis applies two thresholds and if the magnitude is lowers than the first threshold, it is adjusted to zero (made a non-edge). If the magnitude is not below the low threshold, it is made edge. Furthermore, if the magnitude is between the two thresholds, then it is set to zero unless there is a path from this pixel to a pixel with a gradient above the second threshold.

\subsection{Despeckling Techniques}

\subsubsection{Speckle Reducing Anisotropic Diffusion}

The speckle reduction anisotropic diffusion (SRAD) method $(15,16)$ is used directly for suppressing speckle noise in ultrasound and radar images. The SRAD method uses an instant coefficient that is a function of local gradient, magnitude and Laplacian operators. The SRAD method is based on a partial differential equation (PDE) 
Rafati M et al.

that includes the imaging gradient, Laplacian and image intensity. The SRAD equation is presented below (10):

$$
\operatorname{SRAD}\left(U^{\prime}\right)=u t+1=u t+\frac{\Delta t}{4} \operatorname{div}\left(\mathrm{g}\left(\operatorname{ICOV}\left(\mathrm{u}^{\prime}\right)\right) \times \nabla \mathrm{u}^{\prime}\right)
$$

Where $q$ is the diffusion time index, $\Delta \mathrm{t}$ is the time step responsible for the convergence rate of the diffusion process (normally in the range 0.05 to 0.25 ), $g$ ([]) is the diffusion function and is given by Equation 11 and $12(11,12)$.

\begin{tabular}{c}
$G\left(\operatorname{ICOV}\left(u^{\prime}\right)\right)=e-(P)$ \\
$P=\frac{\left(\frac{\operatorname{ICOV}\left(u^{\prime}\right)}{q^{t}}\right)^{2} \cdot-1}{1+\left(q^{t}\right)^{2}}$ \\
\hline
\end{tabular}

Where is the measure of speckle coefficient of variation in a homogenous region of the image.

\subsection{Study Population}

In a cross-sectional design, an examination was performed on ten men with a mean age of $40 \pm 5$ years from September 2012 to March 2013 through random sampling. None of the subjects had a history of hypertension, cardiovascular disease, diabetes mellitus or tobacco abuse (17). All subjects provided an informed consent prior to participation in the study. This study was approved by the ethics committees of Kashan University of Medical Sciences and Beheshti Hospital (Iran; Code ethical approval, 9139; Approval date, 05/08/2012).

\subsection{Ultrasound Studies}

In our study, before ultrasonography, the subjects rested for at least 10 minutes in the supine position until their heart rate and blood pressure reached a steady state. Blood pressure and heart rate were recorded with an oscilloscopic blood manometer $\sigma$ (ALP K2, adult cuff, $\pm 1 \mathrm{mmHg}$, Tokyo, Japan) and a wrist manometer (Microlife, $\pm 3 \mathrm{mmHg}$, Germany) on the left brachial and radial arteries with the subject in a supine position, respectively. The left common carotid of the subject $(2-3 \mathrm{~cm}$ proximal to the bifurcation) and the left brachial artery (3-5 cm upper than elbow) were examined with a Sonoline Antares (Siemens, Bavaria, Germany) ultrasound system equipped with a 5-13 MHz linear transducer. All measurements of both arteries were done by a single operator to eliminate inter-observer variability (19). The audio-video interleaves (AVI) format of the consecutive images of the common carotid and brachial arteries with a frame rate of 43 frames per second was captured from the hard drive and transferred to a PC for post processing. The recording contained 90 frames while the left common carotid and brachial arteries were scanned in the longitudinal direction (Figures 1 and 2). A program was designed using the MATLAB software version 7.01 (Math Software Co., Math Works, USA) to extract consecutive images in the joint picturing expert groups (JPEG) format from the AVI. This software provided the image dimensions (the size of images were $547^{*} 692$ pixel2), image type (B-mode), dynamic range, gray level, depth of focus are $55 \mathrm{~dB}, 0$ to 255 , and $3.5 \mathrm{~cm}$ respectively (18). However, the Sonoline Antares ultrasound imaging system has no quantitative indicator of grayscale mapping and compression curve settings. We think that both are preselected by the machine when probe and application are selected at the start of each examination. Also, when the user selects vascular preset from the menu, the optimum-processing curve (grayscale mapping and compression curve) is probably selected automatically. A vascular preset from the menu was used for all the experiments. Another program was designed in the MATLAB software to apply region of interest (ROI) with a size of approximately $53^{*} 42$ pixel 2 on the intima-media thickness of the posterior wall of common carotid and brachial arteries (19). Then, different edge detections (Sobel, Prewitt, Roberts, Canny, and LOG) and SRAD noise reduction filter were performed on the ROI, separately. Finally, the program measured mean square error (MSE) and peak signal to noise ratio (PSNR) (Figure $3)$. The MSE of the output image was defined as (20):

$$
\mathrm{MSE}=\frac{\sum_{i=1}^{M} \sum_{j=1}^{N} X(i, j)-Y(i, j) .^{2}}{\mathrm{MN}}
$$

Where $X(i, j)$ is the original image, is the next image in sequential images and $\mathrm{MN}$ is the size of the image.

The PSNR was defined (21) as (13):

$$
\mathrm{PSNR}=20 \log _{10} \frac{L^{2}}{\sqrt{\mathrm{MSE}}}
$$

Where $\mathrm{L}$ is the maximum value and MSE is the mean square error.

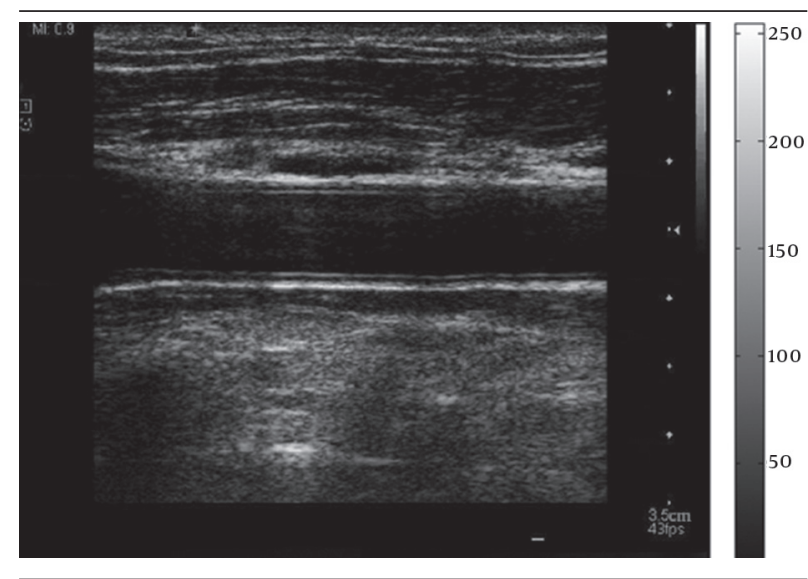

Figure 1. Left Common Carotid Artery Ultrasound Image for One Sample 


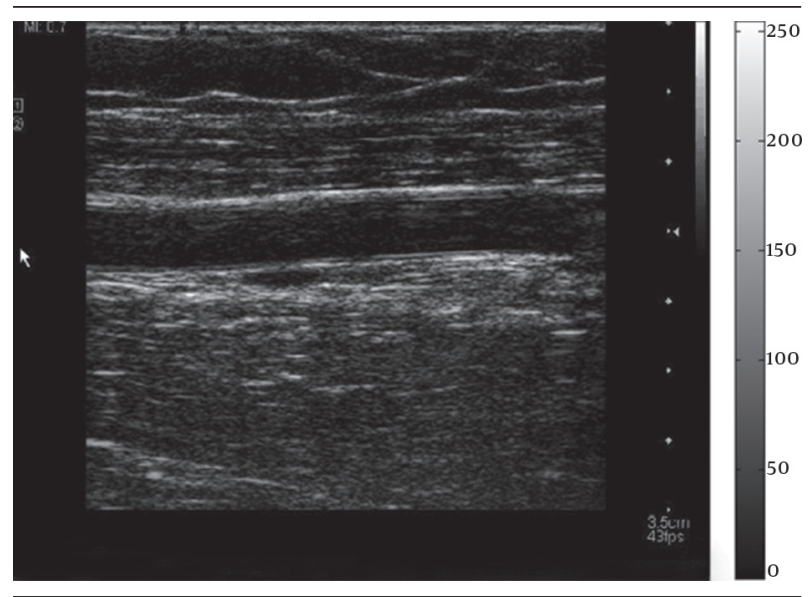

Figure 2. Left Brachial Artery Ultrasound Image for One Sample

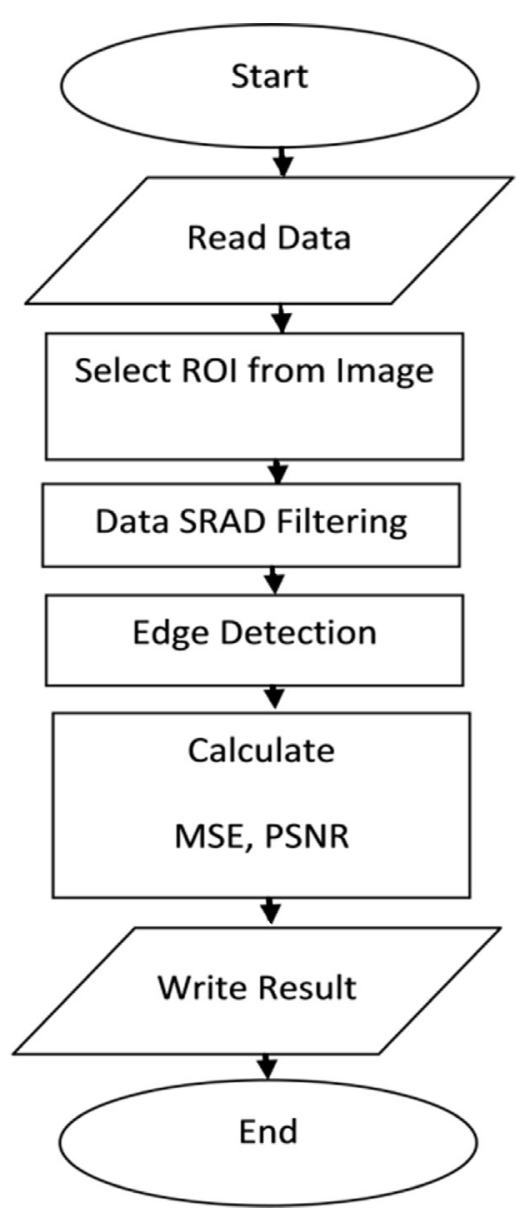

Figure 3. Flowchart of Image Processing Algorithm

\subsection{Statistical Analysis}

All the data are expressed as mean values. The data were tested for normal distribution and homogeneity of variance by the Kolmogorov-Smirnov test (K-S) and Levene's test, respectively. The statistical significance of mean values of MSE and PSNR from edge detections with all filters was assessed by the ANOVA test. The post-hoc test used was the Tukey's test. A P value of $<0.05$ was considered statistically significant. All of the statistical analyses were performed using the SPSS software (SPSS V.11.5, Inc. Chicago, IL). The maximum sample size for the analysis of variance (ANOVA) was estimated from equation 15 (14).

$$
N=\frac{\lambda}{\Delta}
$$

And was sample size, non-centrality parameter respectively $\lambda$ was estimated with a confidence level of $95 \%$ and power test of $80 \%$. The value was calculated from below equation (15):

$$
\Delta=\frac{1}{\sigma^{2}} \sum_{i=1}^{k}\left(\mu_{i}-\mu_{m}\right)^{2}
$$

$$
\mu_{m}=\frac{1}{k} \sum_{j=1}^{k} \mu_{j}
$$

And were number of groups, standard deviation, mean of each group, and overall mean (22).

\section{Results}

The ultrasonic examination of the left common carotid and brachial arteries of ten men (aged $40 \pm 5$ years) with no history of cardiovascular disease, hypertension or diabetes was performed. They had the following characteristics, body mass index (BMI) of $26 \pm 2 \mathrm{~kg} / \mathrm{m}^{2}$; systolic pressure, $127 \pm 19 \mathrm{mmHg}$; diastolic pressure, $80 \pm$ $6 \mathrm{mmHg}$; heart rate (HR), $74 \pm 8 \mathrm{bpm}$ (beat per minute). The results of different edge detections and SRAD denoising filter are summarized in Tables 1, 2, 3 and 4, and Figure 4 and 5 for left common carotid and left brachial arteries, respectively. Tables 1 and 2 show that the lowest values of MSE and highest values of PSNR are achieved via Canny edge detection with de-noising SRAD filter for IMT of left common carotid artery in 90 frames (the temporal resolution was 33 milliseconds). According to the MSE value, there was a significant difference between Canny edge detection status and Sobel, Prewitt, Roberts and LOG edge detections statuses (P-Value < 0.05). Considering, PSNR value, there was a significant difference between Canny edge detection status and all edge detections (Sobel, Prewitt, Roberts and LOG) statuses (P-value $<0.05$ ).

Tables 3 and 4 show that the lowest values of MSE and highest values of PSNR are achieved via Canny edge detection with de-noising SRAD filter for IMT of left brachial artery in 90 frames (almost three cardiac cycles and the time of cardiac cycle was 0.7 seconds in each cycle). According to MSE value, there was a significant difference between Canny edge detection status and all edge detections (So- 
Rafati $M$ et al.

bel, Prewitt, Roberts and LOG) statuses (P-Value < 0.05). Considering, PSNR value, there was a significant difference between Canny edge detection status and all edge detections (Sobel, Prewitt, Roberts and LOG) (P-Value < 0.05).

Figure 4. Ultrasound IMT of Left Common Carotid Artery
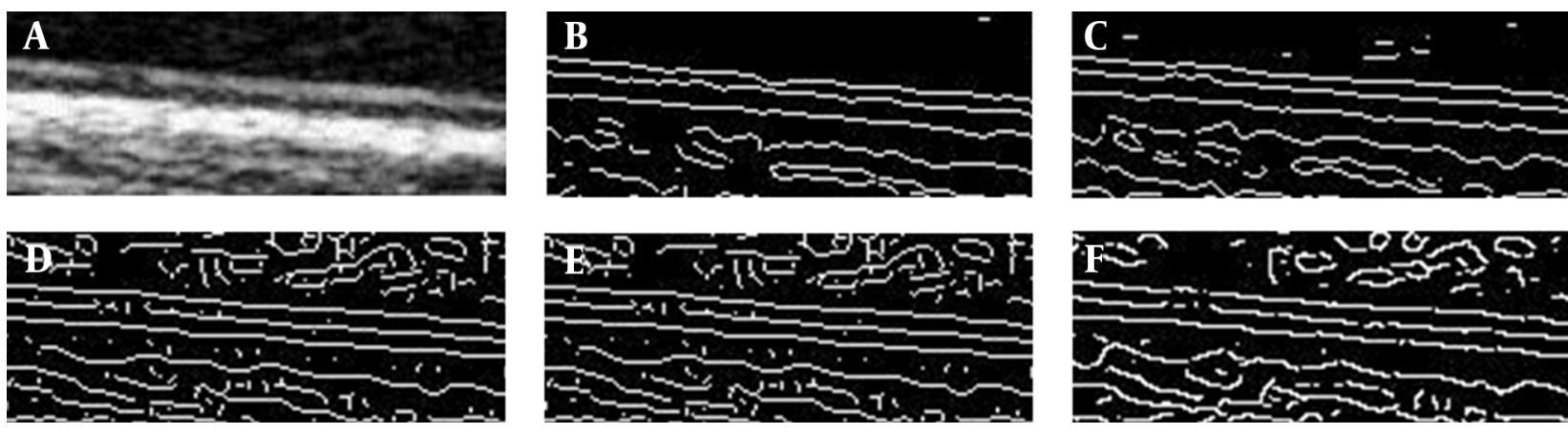

A) Original image, B) SRAD filter-Canny edge detection, C) SRAD filter-Sobel edge detection, D) SRAD filter-Prewitt edge detection, E) SRAD filter-Roberts edge detection, F) SRAD filter-LOG edge detection

Figure 5. Ultrasound IMT of Left Brachial Artery
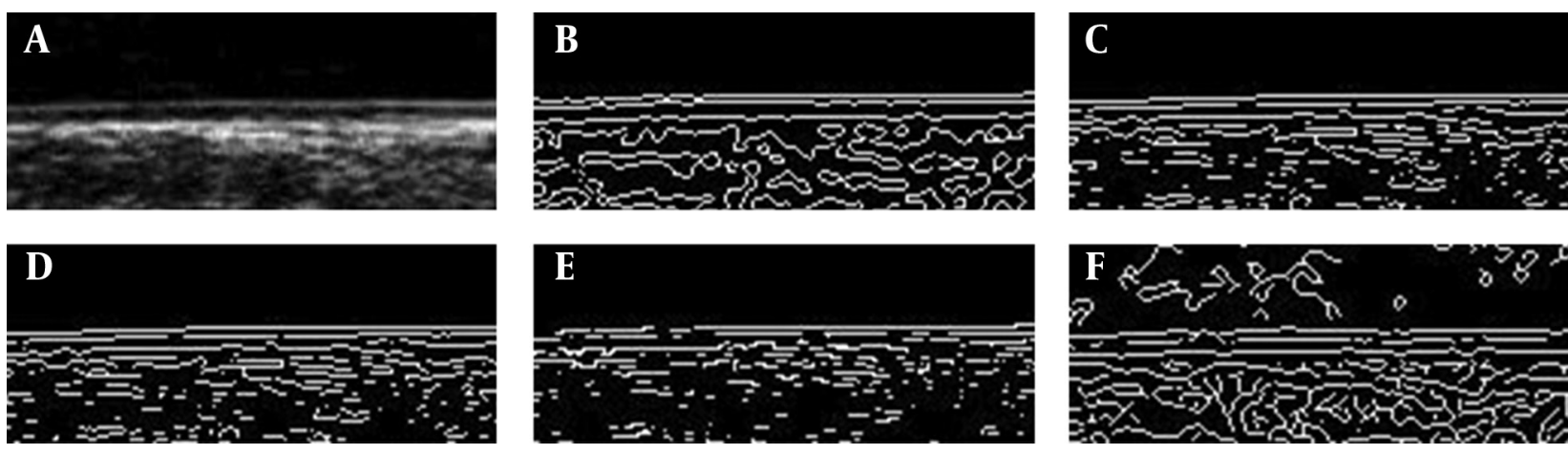

A) Original image, B) SRAD filter-Canny edge detection, C) SRAD filter-Sobel edge detection, D) SRAD filter-Prewitt edge detection, E) SRAD filter-Roberts edge detection, F) SRAD filter-LOG edge detection

Table 1. Mean MSE of SRAD de-Noising Filters and Different Edge Detection in Left Common Carotid Artery (for 90 Frames) for Each Male Candidate ${ }^{a}$

\begin{tabular}{lccccc}
\hline Edge Detection & \multicolumn{5}{c}{ Filters SRAD } \\
\cline { 2 - 6 } & Canny & Sobel & Prewitt & Roberts & LOG \\
\hline MSE-sample 1 & $0.0003 \pm 0.000279$ & $0.0004 \pm 0.000459$ & $0.0014 \pm 0.000796$ & $0.0016 \pm 0.001047$ & $0.0018 \pm 0.001062$ \\
\hline MSE-sample 2 & $0.0005 \pm 0.000269$ & $0.0004 \pm 0.000184$ & $0.0017 \pm 0.000413$ & $0.0018 \pm 0.000506$ & $0.0019 \pm 0.000565$ \\
\hline MSE-sample 3 & $0.0005 \pm 0.000239$ & $0.0004 \pm 0.000179$ & $0.0017 \pm 0.000473$ & $0.0018 \pm 0.000596$ & $0.0020 \pm 0.000584$ \\
\hline MSE-sample 4 & $0.0005 \pm 0.000266$ & $0.0004 \pm 0.000182$ & $0.0016 \pm 0.000448$ & $0.0019 \pm 0.000592$ & $0.0019 \pm 0.000556$ \\
\hline MSE-sample 5 & $0.0005 \pm 0.000244$ & $0.0004 \pm 0.000185$ & $0.0015 \pm 0.000504$ & $0.0018 \pm 0.000562$ & $0.0019 \pm 0.000547$ \\
\hline MSE-sample 6 & $0.0005 \pm 0.00023$ & $0.0004 \pm 0.00017$ & $0.0016 \pm 0.000453$ & $0.0017 \pm 0.000532$ & $0.0020 \pm 0.000532$ \\
\hline MSE-sample 7 & $0.0005 \pm 0.000248$ & $0.0004 \pm 0.000182$ & $0.0017 \pm 0.000498$ & $0.0019 \pm 0.000559$ & $0.0019 \pm 0.000526$ \\
\hline MSE-sample 8 & $0.0005 \pm 0.000266$ & $0.0004 \pm 0.000169$ & $0.0015 \pm 0.000477$ & $0.0018 \pm 0.000585$ & $0.0020 \pm 0.00053$ \\
\hline MSE-sample 9 & $0.0004 \pm 0.000274$ & $0.0004 \pm 0.000173$ & $0.0015 \pm 0.000444$ & $0.0018 \pm 0.000581$ & $0.0020 \pm 0.00057$ \\
\hline MSE-sample 10 & $0.0004 \pm 0.000249$ & $0.0004 \pm 0.000184$ & $0.0016 \pm 0.000482$ & $0.0018 \pm 0.000599$ & $0.0020 \pm 0.000502$ \\
\hline
\end{tabular}

${ }^{\mathrm{a}}$ Data are presented as mean \pm SD. 
Rafati M et al.

Table 2. Mean PSNR of SRAD de-Noising Filters and Different Edge Detection in Left Common Carotid Artery (for 90 Frames) for Each Male Candidate ${ }^{a}$

\begin{tabular}{lccccc}
\hline Edge Detection & \multicolumn{3}{c}{ SRAD Filters } \\
\cline { 2 - 5 } & Canny & Sobel & Prewitt & Roberts \\
\hline PSNR-sample 1 & $85.6530 \pm 4.133385$ & $84.2164 \pm 4.377278$ & $77.3466 \pm 0.000796$ & $76.8923 \pm 2.57703$ & $76.2796 \pm 2.31345$ \\
\hline PSNR-sample 2 & $82.5085 \pm 3.175609$ & $82.7007 \pm 2.45855$ & $76.0447 \pm 0.000413$ & $75.7227 \pm 1.27276$ & $75.5018 \pm 1.35766$ \\
\hline PSNR-sample 3 & $81.8109 \pm 2.52093$ & $82.7018 \pm 2.363957$ & $76.0536 \pm 0.000473$ & $75.8788 \pm 1.57030$ & $75.4361 \pm 1.37893$ \\
\hline PSNR-sample 4 & $81.9928 \pm 3.114101$ & $82.3885 \pm 2.348626$ & $76.2130 \pm 0.000448$ & $75.6858 \pm 1.51850$ & $75.5402 \pm 1.30938$ \\
\hline PSNR-sample 5 & $82.0278 \pm 2.84246$ & $82.5592 \pm 2.281074$ & $76.5037 \pm 0.000504$ & $75.8730 \pm 1.45271$ & $75.4613 \pm 1.31200$ \\
\hline PSNR-sample 6 & $81.7478 \pm 2.637723$ & $82.3815 \pm 1.994495$ & $76.3423 \pm 0.000453$ & $76.0174 \pm 1.44389$ & $75.2075 \pm 1.23105$ \\
\hline PSNR-sample 7 & $81.4933 \pm 2.685306$ & $82.7533 \pm 2.434732$ & $76.1001 \pm 0.000498$ & $75.6047 \pm 1.40218$ & $75.4176 \pm 1.22676$ \\
\hline PSNR-sample 8 & $82.3218 \pm 3.133662$ & $82.4800 \pm 2.125748$ & $76.4409 \pm 0.000477$ & $75.8342 \pm 1.49637$ & $75.3775 \pm 1.23258$ \\
\hline PSNR-sample 9 & $82.7599 \pm 3.451401$ & $82.5319 \pm 2.23298$ & $76.4996 \pm 0.000444$ & $75.9067 \pm 1.54664$ & $75.3388 \pm 1.31783$ \\
\hline PSNR-sample 10 & $82.7074 \pm 3.042031$ & $82.4953 \pm 2.416762$ & $76.3047 \pm 0.000482$ & $75.8680 \pm 1.58317$ & $75.3398 \pm 1.22264$ \\
\hline
\end{tabular}

a Data are presented as mean \pm SD.

Table 3. Mean MSE of SRAD de-Noising Filters and Different Edge Detection in Left Brachial Artery (for 90 Frames) for Each Male Candidate $^{\mathrm{a}}$

\begin{tabular}{lccccc}
\hline Edge Detection & \multicolumn{5}{c}{ SRAD Filters } \\
\cline { 2 - 6 } & Canny & Sobel & Prewitt & Roberts & LOG \\
\hline MSE-sample 1 & $0.0005 \pm 0.000391$ & $0.0009 \pm 0.000612$ & $0.0011 \pm 0.000719$ & $0.0011 \pm 0.000703$ & $0.0012 \pm 0.000537$ \\
MSE-sample 2 & $0.0004 \pm 0.000217$ & $0.0010 \pm 0.000232$ & $0.0011 \pm 0.00028$ & $0.0011 \pm 0.000307$ & $0.0012 \pm 0.000226$ \\
\hline MSE-sample 3 & $0.0005 \pm 0.000219$ & $0.0010 \pm 0.00023$ & $0.0011 \pm 0.000296$ & $0.0011 \pm 0.000294$ & $0.0012 \pm 0.000228$ \\
MSE-sample 4 & $0.0005 \pm 0.000241$ & $0.0010 \pm 0.000235$ & $0.0011 \pm 0.00029$ & $0.0010 \pm 0.000313$ & $0.0012 \pm 0.000252$ \\
\hline MSE-sample 5 & $0.0005 \pm 0.000226$ & $0.0010 \pm 0.000233$ & $0.0011 \pm 0.000292$ & $0.0011 \pm 0.000316$ & $0.0012 \pm 0.000234$ \\
\hline MSE-sample 6 & $0.0005 \pm 0.000232$ & $0.0010 \pm 0.00022$ & $0.0011 \pm 0.000288$ & $0.0011 \pm 0.000313$ & $0.0012 \pm 0.000233$ \\
\hline MSE-sample 7 & $0.0006 \pm 0.000212$ & $0.0010 \pm 0.000234$ & $0.0010 \pm 0.000288$ & $0.0011 \pm 0.000288$ & $0.0012 \pm 0.000227$ \\
\hline MSE-sample 8 & $0.0005 \pm 0.000218$ & $0.0010 \pm 0.000232$ & $0.0011 \pm 0.000299$ & $0.0011 \pm 0.0003$ & $0.0012 \pm 0.000239$ \\
\hline MSE-sample 9 & $0.0005 \pm 0.000221$ & $0.0010 \pm 0.000244$ & $0.0011 \pm 0.000288$ & $0.0011 \pm 0.000352$ & $0.0012 \pm 0.000224$ \\
\hline MSE-sample 10 & $0.0005 \pm 0.000229$ & $0.0010 \pm 0.000228$ & $0.0011 \pm 0.000288$ & $0.0010 \pm 0.000352$ & $0.0012 \pm 0.000241$ \\
\hline
\end{tabular}

${ }^{\mathrm{a}}$ Data are presented as mean \pm SD.

\begin{tabular}{lccccc}
\hline \multicolumn{7}{l}{ Table 4. Mean PSNR of SRAD de-Noising Filters and Different Edge Detection in Left Brachial Artery (for 90 frames) for Each Man ${ }^{\mathrm{a}}$} \\
\hline \multicolumn{7}{l}{ Filters } & \multicolumn{4}{c}{ SRAD } & & \\
\hline Edge Detection & Canny & Sobel & Prewitt & Roberts & LOG \\
\hline PSNR-sample 1 & $82.4307 \pm 2.763456$ & $79.1419 \pm 2.116285$ & $78.4712 \pm 2.122997$ & $78.3275 \pm 2.088506$ & $77.4947 \pm 1.482641$ \\
\hline PSNR-sample 2 & $82.2781 \pm 2.374491$ & $78.2678 \pm 1.036241$ & $77.9120 \pm 1.185247$ & $77.9116 \pm 1.337343$ & $77.4947 \pm 1.482641$ \\
PSNR-sample 3 & $81.6568 \pm 2.272825$ & $78.1484 \pm 1.020246$ & $77.8382 \pm 1.244113$ & $78.0146 \pm 1.263799$ & $77.4280 \pm 0.855661$ \\
\hline PSNR-sample 4 & $81.6019 \pm 2.545126$ & $78.3120 \pm 1.064856$ & $77.7409 \pm 1.18226$ & $78.1780 \pm 1.393633$ & $77.4368 \pm 0.931652$ \\
\hline PSNR-sample 5 & $81.5119 \pm 2.324907$ & $78.1584 \pm 1.041796$ & $77.9120 \pm 1.22399$ & $78.0013 \pm 1.352399$ & $77.4798 \pm 0.887956$ \\
\hline PSNR-sample 6 & $81.6738 \pm 2.464557$ & $78.0666 \pm 0.968866$ & $77.8407 \pm 1.194547$ & $78.1033 \pm 1.374629$ & $77.4359 \pm 0.863877$ \\
\hline PSNR-sample 7 & $80.9981 \pm 2.103223$ & $78.1372 \pm 1.035725$ & $77.7073 \pm 1.176266$ & $77.9922 \pm 1.2899$ & $77.3818 \pm 0.850334$ \\
\hline PSNR-sample 8 & $81.6211 \pm 2.327975$ & $78.1975 \pm 1.053496$ & $77.9091 \pm 1.250404$ & $78.0517 \pm 1.332564$ & $77.4297 \pm 0.894732$ \\
\hline PSNR-sample 9 & $82.2185 \pm 2.490667$ & $78.3140 \pm 1.116204$ & $77.9093 \pm 1.194592$ & $78.0561 \pm 1.550084$ & $77.3518 \pm 0.825746$ \\
PSNR-sample 10 & $81.8289 \pm 2.530808$ & $78.2992 \pm 1.032096$ & $77.7881 \pm 1.180824$ & $78.2153 \pm 1.595926$ & $77.3825 \pm 0.89572$ \\
\hline
\end{tabular}

\footnotetext{
${ }^{\mathrm{a}}$ Data are presented as mean \pm SD.
} 


\section{Discussion}

Nowadays, intima-media thickness (IMT) of common carotid artery is considered as an important value for prediction of cardiovascular diseases. Therefore, precise measurement of IMT is important to assess the risk of cardiovascular events or to evaluate their progress (1). Moreover, Weidinger et al. showed that assessment of brachial artery that is less prone to symptomatic obstructive disease compared to other arterial beds (carotid, femoral) might have some potential advantages. First, changes of function and morphology can be detected in the same artery. Second, the pattern of wall thickening in the brachial artery is rather diffuse compared to carotid artery and may be a more sensitive indicator of long-term systemic exposure to risk factors (23). Therefore, we studied these arteries (CCA and BA) in our research. Edge detection in ultrasonic images is important for recognition of IMT in CCA and BA. Edge detection operator is a mutation in the nature of the image to test the edge. Conventionally, the edge is detected according to algorithms like Sobel, Roberts, Prewitt, Canny and LOG (Laplacian of Gaussian) operators (4). The use of the Sobel edge detector is somewhat difficult compared to the Prewitt edge detector. Prewitt edge detector is slightly simpler to implement computationally than the Sobel detector. However, it tends to cause slightly noisier results (24). Roberts edge operator is one of the oldest and simplest edge detectors in digital imaging (25). It is still used frequently in hardware implementations where simplicity and speed are dominant factors (25). The disadvantages of these operators (Sobel, Prewitt and Roberts) are sensitivity to noise in detection of the edges and their orientations. The increase in the noise of the image will eventually degrade the magnitude of the edges $(5,26)$. The LOG is often applied to an image that has first been smoothed with something approximating a Gaussian-smoothing filter in order to reduce its sensitivity to noise (27). The disadvantage is that it reduces the accuracy in finding out the orientation of edges and malfunctions the corners and curves, where the gray level intensity function varies (27). Canny has three criteria for the evaluation of performance of edge detection (24). The first and most obvious is low error rate. It is important that edges occurring in images should not be missed and that there should be no responses to non-edges. The second criterion is that the edge points should be well localized. In other words, the distance between the edge pixels as found by the detector and the actual edge should be at its minimum. The third criterion is to have only one response to a signal edge (25). This study showed that Canny edge detection with SRAD filter is better than several commonly used edge detections with SRAD filter in terms of speckle suppression and details preservation in common carotid and brachial arteries ultrasound images. The present study is in agreement with many other studies $(23,28)$. Using the automatic method for measuring the intimamedia thickness in B-mode ultrasound images was easy and highly reproducible (29). Additionally, the duration of image processing was considerably reduced, and the variation in results seen with manual tracing observers was IMT changes in the common carotid artery (29). In this study, we used the computerized analysis method to detect the dynamic mechanical properties of both artery (carotid and brachial arteries) walls in three cardiac cycles. A few investigations have shown that changes in IMT occur during a cardiac cycle $(30,31)$. Selzer et al. showed that common carotid was lower during the peak systolic period compared to the end of the diastolic by an average of 5.3\% in 24 individuals (32). Another study showed that the average change in carotid IMT during the cardiac cycle was $0.041 \mathrm{~mm}$ (33). Hence, the present study assessed different edge detections with SRAD de-noising for ultrasound images of common carotid and brachial arteries in 90 frames (almost three cardiac cycles). Moreover, reduction of noise was utilized on ultrasound images of IMT in both arteries (carotid and brachial arteries). Finally, our study may provide more comprehensive information than other previous studies in which edge detection was done only on one frame or one artery. However, a limitation of this study is that SRAD filtration is a time consuming process for ultrasound images. Another limitation of the current study was the translational movement of common carotid and brachial arteries, which are related to probe movement during scanning and suppression of artery pulsating movements. However, we tried to make these movements as low as possible. According to IMT changes during the cardiac cycle, the present study assessed different edge detections with SRAD de-noising filter for ultrasound images of common carotid and brachial arteries in 90 frames (almost three cardiac cycles). Based on the result, by measuring the MSE and PSNR, this study showed that Canny edge detection with SRAD filter is better than other edge detections used with SRAD filter in terms of speckle suppression and details preservation in common carotid and brachial arteries ultrasound images.

\section{Acknowledgements}

The Authors declare that they have no conflicts of interest and they do not have a financial relationship with the organization that sponsored the research. 


\section{References}

1. Polak JF, Pencina MJ, Meisner A, Pencina KM, Brown LS, Wolf PA, et al. Associations of carotid artery intima-media thickness (IMT) with risk factors and prevalent cardiovascular disease: comparison of mean common carotid artery IMT with maximum internal carotid artery IMT. J Ultrasound Med. 2010;29(12):1759-68.

2. Gutierrez MA, Pilon PE, Lage SG, Kopel L, Carvalho RT, Furuie SS. Automatic measurement of carotid diameter and wall thickness in ultrasound images. Computers cardiol. 2002:359-62.

3. del Sol AI, Moons KG, Hollander M, Hofman A, Koudstaal PJ, Grobbee $\mathrm{DE}$, et al. Is carotid intima-media thickness useful in cardiovascular disease risk assessment? The Rotterdam Study. Stroke. 2001;32(7):1532-8.

4. Hareesh KS, Narendra VG. Study and comparison of various image edge detection techniques used in quality inspection and evaluation of agricultural and food products by computer vision. Int Agric Biol Eng J. 2011:83-90.

5. Rashmi S, Kumar M, Saxena R. Algorithm and technique on various edge detection: A survey. Signal Image P. 2013.

6. Fernandez CA. Finding out tendencies in speckle noise reduction in ultrasoud images. Expert Systems App. 2009:7786-97.

7. Chandrasekar C, Karthikeyan K. Speckle Noise Reduction of Medical Ultrasound Images using Bayesshrink Wavelet Threshold. Int Computer App J. 2011;22(9):8-14.

8. Sun Q, Hossack JA, Tang J, Acton ST. Speckle reducing anisotropic diffusion for 3D ultrasound images. Comput Med Imaging Graph. 2004;28(8):461-70.

9. Yongjian Y, Acton ST. Speckle reducing anisotropic diffusion. IEEE Ton Image Processing. 2002;11(11):1260-70.

10. Nixon MS, Aguado AS. Low-level feature extraction. In: 3 editor. Including edge detection.. Jordan Hill: Oxford: Elsivier; 2008. pp. 115-79.

11. Muthukrishnan R, Radha M. Edge detection techniques for image segmentation. Int Computer S I Technol J. 2011;3:259-67.

12. Heath M, Sarkar S, Sanocki T, Bowyer K. Comparison of Edge detectors. Comput vision Image Und. 1998:38-54.

13. Haider W, Malik MS, Raza MS, Wahab A, Khan IA, Zia U, et al. A hy brid method for edge continuity based on pixel neighbers patterns (PNPA) for remote sensing satellite images. Int'l Commun Network Syst S J. 2012;5:624-30.

14. Ilic S, Timotijevic S, Lazovic D, Spalevic P, Veljovic A. Comparison of different methods for extracting bone cross-sectional geometric properties from CT images. Serbian Electr Eng. 2013; 10(2):247-60.

15. El-said S, Azar AT. Speckles suppression techniques for ultrasound images. Medic Imaging Radiat S J. 2012;43:200-13.

16. Geethalakshmi SN, Suguna J. Hybrid models for denoising ultrasonic images. Global Res Computer Sci J. 2010:38-45.

17. Rafati M, Mokhtari DM, Saberi H. The Effect of Cuff Occlusion Pro- tocols on Radial Strain and Arterial Haemodynamics. Ultrasound 2009;17(3):144-9.

18. Touboul PJ, Hennerici MG, Meairs S, Adams H, Amarenco P, Bornstein N, et al. Mannheim Carotid Intima-Media Thickness Consensus. Cerebrovas Dis. 2007;23(1):75-80.

19. Golemati S, Sassano A, Lever MJ, Bharath AA, Dhanjil S, Nicolaides AN. Carotid artery wall motion estimated from b-mode ultrasound using region tracking and block matching. Ultrasound Med Biol. 2003;29(3):387-99.

20. Shubhangi DC, Raghavendra SC, Hiremath PC. Edge detection of femur bones in x-ray images - A comparative study edge detectors. Intl Computer Applications J. 2012:13-6.

21. Lin C, Wang MS. A Vision Based Top-View Transformation Model for a Vehicle Parking Assistant. Sensors. 2012;12(12):4431-46.

22. Chow SC, Shao J, Wang H. Sample size calculations in clinical research. 2 ed. New York; 2003. pp. 79-81.

23. Weidinger F, Frick M, Alber HF, Ulmer H, Schwarzacher SP, Pachinger $\mathrm{O}$. Association of wall thickness of the brachial artery measured with high-resolution ultrasound with risk factors and coronary artery disease. The Americ Cardiol J. 2002;89(9):1025-9.

24. Canny J. A computational approach to edge detection. IEEE Trans Pattern Anal Mach Intell. 1986;8(6):679-98.

25. Solomon C, Breckon T. Fundamentals of Digital Image Processing.; 2011.

26. Chen YB, Chen OTC. Image Segmentation Method Using Thresholds Automatically Determined from Picture Contents. EURASIP. 2009;2009:1-15.

27. Ghoshal D, Singh Bhadouria V, Pande S. A Study on Edge Marking Scheme of Various Standard Edge Detectors. Int Computer App J. 2012;44(9):33-7.

28. Damodaran N, Ramamurthy S, Velusamy S, Kanakaraj M G. Speckle Noise Reduction in Ultrasound Biomedical B-Scan Images Using Discrete Topological Derivative. Ultrasound Med Biol. 2012;38(2):276-86.

29. Wendelhag I, Liang Q, Gustavsson T, Wikstrand J. A New Automated Computerized Analyzing System Simplifies Readings and Reduces the Variability in Ultrasound Measurement of IntimaMedia Thickness. Stroke.1997;28(11):2195-200.

30. Kanai H, Koiwa Y. Real-time velocimetry for evaluation of change in thickness of arterial wall. Ultrasonics. 2000;38(1-8):381-6.

31. Boutouyrie P, Germain DP, Tropeano AI, Laloux B, Carenzi F, Zidi $\mathrm{M}$, et al. Compressibility of the Carotid Artery in Patients With Pseudoxanthoma Elasticum. Hypertension. 2001;38(5):1181-4.

32. Selzer R. Improved common carotid elasticity and intima-media thickness measurements from computer analysis of sequential ultrasound frames. Atherosclerosis. 2001;154(1):185-93.

33. Polak JF, Johnson C, Harrington A, Wong Q, O'Leary DH, Burke $\mathrm{G}$, et al. Changes in Carotid Intima-Media Thickness During the Cardiac Cycle: The Multi-Ethnic Study of Atherosclerosis. Americ Heart Association J. 2012;1(4):e001420. 